\title{
Tratamento com Hormônio de Crescimento em Crianças com Baixa Estatura Nascidas Pequenas para a Idade Gestacional
}

revisão

\section{Margaret C. S. BoguszeWSKI \\ CÉSAR LUIz BOGUSZEWSKI}

Unidade de Endocrinologia Pediátrica do Departamento de

Pediatria (MCSB); Serviço de

Endocrinologia e Metabologia do Paraná do Departamento de

Clínica Médica (CLB) da Universidade Federal do Paraná, Curitiba, PR, Brasil.
Aproximadamente $10 \%$ das crianças nascidas pequenas para a idade gestacional (PIG) não apresentam recuperação pós-natal do crescimento e permanecem com baixa estatura na infância e na vida adulta. Em alguns casos é possível identificar alterações no eixo GH/IGF-1. Em outros, a avaliação com os recursos diagnósticos disponíveis até o momento é normal. $\mathrm{O}$ tratamento com GH tem mostrado resultados promissores com recuperação do crescimento e da altura adulta dentro do esperado para a população. Nesta revisão são apresentados resultados do tratamento com $\mathrm{GH}$ em crianças com baixa estatura nascidas PIG ressaltando a necessidade do tratamento precoce. (Arq Bras Endocrinol Metab 2008; 52/5:792-799)

Descritores: Hormônio do crescimento; Pesqueno para a idade gestacional; Baixa estatura

\section{ABSTRACT}

Growth Hormone Therapy for Short Children Born Small for Gestational Age. Approximately $10 \%$ of all children born small for gestational age (SGA) fail to achieve sufficient catch-up growth and remain with short stature throughout childhood and adult life. Abnormalities of the GH/IGF-1 axis are not always identified. Several studies have demonstrated that $\mathrm{GH}$ is an effective and well-tolerated therapy and most children will reach a normal adult height. In this review, it can be seen the encouraging results of $\mathrm{GH}$ treatment in growthretarded children born SGA highlighting the benefits of early treatment. (Arq Bras Endocrinol Metab 2008; 52/5:792-799)

Keywords: Growth hormone; Small for gestational age; Short stature

\section{INTRODUÇÃO}

Cegundo a Organização Mundial da Saúde (OMS), 8\% a 26\% das crianças $\checkmark$ nascidas em diferentes regiões do mundo apresentam peso ao nascimento abaixo de $2.500 \mathrm{~g}$, entre bebês nascidos a termo e prematuros. Nos países desenvolvidos predominam os prematuros. Já nos países em desenvolvimento a freqüência maior é de bebês nascidos pequenos para a idade gestacional (PIG). Estima-se que 2,3\% a 10\% de todos recém-nascidos sejam PIG. Considerando a estimativa de 2,3\%, é esperado que nasçam aproximadamente 95 mil bebês PIG nos Estados Unidos por ano. No mesmo período, estima-se que nasçam aproximadamente 1.200 crianças com deficiência de hormônio de crescimento $(\mathrm{GH})(\mathrm{l})$. Como um grupo, as crianças nascidas PIG apresentam maior morbi e mortalidade perinatal e maior risco de baixa estatura desde a infância até a vida adulta (2). Estes números mostram a importância do acompanhamento regular das crianças nascidas PIG.
Recebido em 3/5/2008

Aceito em 20/5/2008 
Por muito tempo, diferentes critérios foram utilizados para definir crianças nascidas PIG, o que resultava grupos bastante heterogêneos (peso de nascimento abaixo do $10^{\circ}$ ou $5^{\circ}$ percentil para a idade gestacional, peso abaixo de 2.500 g para a idade gestacional maior ou igual a 37 semanas, peso e/ou comprimento mais que dois desvios-padrão (DP) abaixo da média para idade gestacional) (3). Em 2001, uma reunião de consenso do International Small for Gestational Age Advisory Board definiu como PIG apenas as crianças nascidas com peso e/ou comprimento 2 ou mais DP abaixo da média para a idade gestacional (4). Esta definição foi endossada pelas sociedades internacionais de endocrinologia pediátrica e pela Growth Hormone Research Society (5).

\section{CRESCIMENTO PÓS-NATAL}

Considera-se que uma criança está em fase de recuperação do crescimento ou apresentando catch-up quando a velocidade de crescimento ( $\mathrm{cm} / \mathrm{ano})$ está acima da média para a idade e sexo (1). A avaliação do crescimento pela altura esperada para a idade (por exemplo, baixa estatura ou não recuperação do crescimento se estatura menor que -2 DP) não leva em consideração o potencial genético da criança. A maioria das crianças nascidas PIG apresenta recuperação do crescimento e normalização da altura nos dois primeiros anos de vida, visto que o início da recuperação costuma ser precoce, ao redor das 12 semanas de vida (6). Uma pequena porcentagem de crianças apresenta recuperação do crescimento um pouco mais lenta, com normalização da altura ao redor dos 4 anos de idade. Entretanto, $10 \%$ a $15 \%$ das crianças nascidas PIG não normalizam o crescimento nos primeiros anos de vida e chegam à idade adulta com estatura abaixo da esperada $(2,7,8)$. Chaussain, Colle e Ducret (9) demonstraram que a estatura adulta ficou em média $7,5 \mathrm{~cm}$ abaixo do alvo familiar em pacientes do sexo masculino e $9,6 \mathrm{~cm}$ no sexo feminino. Carrascosa e cols. (10) demonstraram que crianças nascidas PIG que persistiram com baixa estatura entre os 2 e os 8 anos de idade chegaram à idade adulta com estatura $1 \mathrm{DP}$ ou mais abaixo da estatura média familiar. $\mathrm{O}$ risco relativo de baixa estatura aos 18 anos de idade em jovens nascidos PIG comparados com jovens nascidos adequados para a idade gestacional (AIG) foi 7,1 vezes maior quando PIG foi definido pelo talhe de nascimento e 5,2 vezes maior quando definido pelo peso de nascimento (2). Em relação ao crescimento na puberdade, as crianças nascidas PIG parecem iniciar a puberdade com idade cronológica dentro dos limites da normalidade para crianças sem baixa estatura, porém um pouco mais precoce do que as crianças com baixa estatura idiopática. A intensidade do estirão de crescimento durante a puberdade é menor nestas crianças levando em consideração a idade relativamente mais precoce em que ocorre $(8,11)$. O ganho de altura da puberdade até a idade adulta ficou em torno de 0,5 DP (12).

\section{EIXO GH/IGF-1}

A relação entre baixa estatura, secreção de $\mathrm{GH}$ e níveis de IGF-1 nas crianças nascidas PIG ainda é motivo de discussões (13-16). Como um grupo, crianças com baixa estatura nascidas PIG apresentam secreção espontânea de GH diminuída nas 24 horas com padrão anormal de secreção comparado com crianças nascidas AIG (13). A avaliação individual demonstrou casos de secreção normal até deficiência de $\mathrm{GH}(13,17)$. Quanto aos níveis séricos de IGF- 1 , níveis diminuídos foram descritos $(17,18)$, porém alguns autores sugerem que certo grau de resistência ao GH e IGF-1 possa ocorrer nestas crianças (19). Völkl e cols. demonstraram I DP abaixo da média em crianças com baixa estatura nascidas PIG, em concentrações de IGF-1, a despeito da resposta normal do GH nos testes provocativos (20).

\section{TRATAMENTO COM HORMÔNIO DE CRESCIMENTO}

O tratamento com hormônio de crescimento para crianças com baixa estatura nascidas PIG visa a aumentar a velocidade de crescimento e normalizar a altura durante a infância, a adolescência e a vida adulta. Em 26 de julho de 2001, a Food and Drug Administration (FDA), nos Estados Unidos, aprovou o uso de hormônio de crescimento sintético na dose de $66 \mu \mathrm{g} / \mathrm{kg} / \mathrm{dia}$, equivalente a aproximadamente $0,06 \mathrm{mg} / \mathrm{kg} / \mathrm{dia}$ ou $0,2 \mathrm{UI} / \mathrm{kg} / \mathrm{dia}$, para crianças nascidas PIG com estatura abaixo de -2.0 DP e que não apresentaram recuperação do crescimento até os 2 anos de idade (21). Na Europa, a European Medicines Evaluation Agency (EMEA), em 2003, aprovou o uso na dose de $35 \mu \mathrm{g} /$ $\mathrm{kg} /$ dia, equivalente a aproximadamente a $0,03 \mathrm{mg} /$ $\mathrm{kg} /$ dia ou $0,1 \mathrm{UI} / \mathrm{kg} /$ dia, para crianças nascidas PIG com estatura abaixo de $-2,5$ DP e 1 DP abaixo do esperado para o alvo genético que não apresentaram recuperação do crescimento até os 4 anos de idade (22). 
As duas indicações foram independentes da secreção de $\mathrm{GH}$ e de testes provocativos.

\section{Efeitos a curto e médio prazo do tratamento com GH}

A primeira descrição do uso de GH para o tratamento da baixa estatura em crianças nascidas PIG data de 1970, quando 18 crianças foram tratadas com hormônio de crescimento extraído de hipófises humanas (23). Neste período, a disponibilidade da medicação era pequena e poucos estudos foram realizados (24-26). No final da década de 1980 e início dos anos 1990, com a comercialização do hormônio de crescimento sintético a partir de 1985, tiveram início os estudos com maior número de pacientes e com períodos mais longos de tratamento. Também foi possível avaliar o efeito de diferentes doses de GH. Apesar de os grupos tratados serem bastante heterogêneos, com diferentes critérios utilizados para definir PIG, as mais diversas concentrações de GH durante testes provocativos e a ausência de grupo-controle em alguns estudos, os resultados foram promissores com aumento da velocidade de crescimento e melhora da estatura (27-33). O efeito dose-dependente nos primeiros anos de tratamento se tornou evidente $(29,30,34,35)$. Um ganho médio na altura de 1,72 DP foi demonstrado após três anos de tratamento nas crianças recebendo $0,1 \mathrm{UI} / \mathrm{kg} /$ dia de $\mathrm{GH}$, comparado com um ganho médio de 2,54 DP nas crianças recebendo $0,2 \mathrm{UI} / \mathrm{kg} /$ dia. Ao final de três anos de tratamento, todas as crianças recebendo a maior dose tinham atingido altura dentro do seu potencial genético. No mesmo período, as crianças do grupo-controle ganharam apenas $0,14 \mathrm{DP}$ na altura (35). Sas e cols. (36) avaliaram os efeitos de cinco anos de tratamento com doses de 3 e $6 \mathrm{UI} / \mathrm{m}^{2} /$ dia de GH. Os autores demonstraram aumento médio de 2,6 DP na altura no grupo que recebeu a maior dose e de 2,2 DP no grupo com a dose menor, porém sem diferença significativa entre os grupos. O efeito dose-dependente foi significativo apenas entre as crianças que permaneceram pré-púberes durante todo o período de tratamento. A resposta não foi diferente entre deficientes e não-deficientes de $\mathrm{GH}$ e a puberdade teve início em idade normal. Houve aumento médio de 9,1 a $14 \mathrm{~cm}$ na previsão de altura adulta após cinco anos de tratamento, sendo maior naqueles com maior dose de GH.

Mais recentemente, Argente e cols. (37) demonstraram os efeitos do tratamento precoce com 0,06 $\mathrm{mg} / \mathrm{kg} /$ dia de $\mathrm{GH}$ em crianças com 5 anos de idade ou menos e que não apresentaram recuperação espontânea do crescimento. O ganho médio de altura em dois anos de tratamento foi de 2,1 DP e as crianças com menos idade apresentaram melhor resposta. Não houve avanço significativo da idade óssea no período. Os autores sugerem que tratamento precoce com doses altas de GH pode trazer benefícios em longo prazo.

\section{Altura adulta final}

$\mathrm{O}$ primeiro relato de altura adulta obtida com tratamento com GH em crianças nascidas PIG foi feito por Ranke e Lindberg, em 1996, utilizando informações do banco de dados do KIGS (Kabi International Growth Study) (38). Os autores encontraram aumento médio de 1 DP na estatura próxima da final em 16 jovens tratados com dose média de $0,7 \mathrm{UI} / \mathrm{kg} /$ semana pelo período médio de 4,3 anos, apesar de o início tardio do tratamento, em média aos 12,7 anos. Já Coutant e cols. (39) não demonstraram melhora na estatura final comparada com a altura de jovens sem tratamento. Porém, os pacientes receberam doses baixas de $\mathrm{GH}(0,4 \mathrm{UI} /$ $\mathrm{kg} / \mathrm{semana}$ ) e também iniciaram o tratamento com idade cronológica avançada, acima de 12 anos. Resultados semelhantes foram descritos por Zucchini e cols., que consideraram PIG as crianças nascidas com peso abaixo do $10^{\circ}$ percentil para a idade gestacional e trataram com $20 \mathrm{UI} / \mathrm{m}^{2} /$ semana (aproximadamente $0,1 \mathrm{UI} /$ $\mathrm{kg} /$ dia) apenas as crianças com pico de $\mathrm{GH}$ durante teste provocativo abaixo de $8 \mu \mathrm{g} / \mathrm{l}(40)$.

A idade e a estatura no início do tratamento são consideradas fatores importantes na resposta ao tratamento. Carel e cols. (12) trataram um grupo de crianças nascidas PIG já em puberdade ou com idade cronológica e óssea próximas do início da puberdade. O tempo médio de tratamento foi de 2,7 anos e não houve intervenção na evolução da puberdade. Os autores observaram que as crianças que não receberam GH aumentaram apenas $0,5 \mathrm{DP}$ na altura do início da puberdade até a altura adulta final, reforçando o conceito de que crianças nascidas PIG não apresentam recuperação espontânea do crescimento durante a puberdade. Já as crianças que receberam GH na dose de $0,67 \mathrm{mg} /$ $\mathrm{kg} /$ dia aumentaram a altura em $1,1 \mathrm{DP}$, evidenciando ganho real com o tratamento de $0,6 \mathrm{DP}$, o que corresponde a $+4 \mathrm{~cm}$ ou $18 \%$ mais crescimento no grupo tratado. A idade óssea era atrasada e avançou durante o tratamento, fazendo que o ganho em altura ficasse menor que o esperado no início do tratamento. Os autores 
sugeriram que a associação com análogo de $\mathrm{GnRH}$ poderia potencializar o ganho de altura quando o tratamento com GH é feito durante a puberdade.

Em um dos estudos mais longos de tratamento com GH (41), 54 crianças com baixa estatura nascidas PIG foram tratadas pelo período médio de 7,8 $\pm 1,7$ anos; $85 \%$ delas ficaram com altura adulta acima de -2 DP e $98 \%$ atingiram uma altura dentro do esperado para o alvo genético. O ganho médio foi de 1,8 DP de altura (aproximadamente $12 \mathrm{~cm}$ para meninos e $11 \mathrm{~cm}$ para meninas) nas crianças que receberam $0,03 \mathrm{mg} /$ $\mathrm{kg} /$ dia e de 2,1 DP (aproximadamente $14 \mathrm{~cm}$ para meninos e $13 \mathrm{~cm}$ para meninas) nas que receberam o dobro da dose. Não houve relação entre a dose utilizada e o ganho total de altura, indicando que o efeito dosedependente perde força durante o tratamento mais longo. O início da puberdade ocorreu ao redor dos 11,7 anos nos meninos com incremento de 27 a $30 \mathrm{~cm}$ até altura adulta. Nas meninas, a puberdade teve início ao redor de 10,8 anos com ganho ao redor de $19 \mathrm{~cm}$ até a altura adulta. Apesar de não ocorrem diferenças significativas, os autores observaram que a idade óssea, ao redor de 0,5 anos menor que a idade cronológica no início do tratamento, ficou um ano acima da cronológica após cinco anos de tratamento.

Mais recentemente, Dahlgren e Wikland demonstraram a influência do crescimento antes da puberdade sobre a altura adulta final (42). Foram tratadas com GH na dose de $0,1 \mathrm{UI} / \mathrm{kg} /$ dia e ficaram em média 1,3 DP $(9 \mathrm{~cm})$ acima da altura prevista no início do tratamento 77 crianças. Entre as crianças tratadas por dois anos ou mais antes do início da puberdade, o ganho médio foi de 1,7 DP. Já nas que tiveram um período de tratamento menor que dois anos, antes da puberdade, o ganho foi menor, de 0,9 DP. O ganho de altura obtido com o tratamento antes da puberdade foi mantido até a altura adulta. Já o tratamento durante a puberdade preservou a altura, sem que ocorresse aumento no DP da altura. Os autores sugerem que quanto menor a idade, a altura e o peso no início do tratamento, melhor será a altura adulta.

Poucos estudos são disponíveis sobre a altura final de pacientes nascidos PIG com baixa estatura no início da puberdade e tratados com GH e bloqueio da puberdade. Van Gool e cols. (43) avaliaram 17 adolescentes tratados durante três anos com terapia combinada de $\mathrm{GH}$ + análogo de GnRH e compararam com o crescimento de 15 adolescentes não-tratados. A idade média no momento da interrupção do tratamento foi de 14,9 anos, visto que neste momento o aumento na previsão de altura adulta era de $9,3 \mathrm{~cm}$ no grupo tratado e apenas $1,2 \mathrm{~cm}$ no grupo-controle. Entretanto, ao atingir a altura final, o ganho médio na altura foi de $4,9 \mathrm{~cm}$ no grupo tratado comparado ao grupo não-tratado e a altura final não foi diferente entre os grupos. Os autores consideraram o ganho pequeno para a intensidade e o custo do tratamento.

\section{Tratamento descontínuo com GH}

Crianças com baixa estatura de diferentes etiologias apresentam velocidade de crescimento maior no primeiro ano de tratamento com $\mathrm{GH}$ com diminuição nos anos subseqüentes. Também é comum a desaceleração do crescimento após a parada do tratamento com GH. Esta desaceleração foi demonstrada em crianças nascidas PIG após a parada do tratamento com GH $(34,44$, 45). Alguns estudos avaliaram se o tratamento intermitente poderia potencializar a resposta ao tratamento, tendo em vista que um novo aumento de velocidade de crescimento poderia se somar à resposta inicial do tratamento a cada vez que o tratamento fosse retomado.

Simon e cols. (46) avaliaram os benefícios do tratamento descontínuo na dose de $67 \mu \mathrm{g} / \mathrm{kg} / \mathrm{dia}$. As crianças foram acompanhadas durante quatro anos. Um grupo recebendo GH ano sim, ano não. Outro grupo recebeu GH dois anos consecutivos seguidos de dois anos sem GH. Ambos os grupos apresentaram aumento da velocidade de crescimento durante os períodos de tratamento, porém acompanhado de importante diminuição da velocidade de crescimento nos períodos sem GH. Apesar da boa velocidade de crescimento durante o tratamento, a interrupção anual do tratamento não levou a maior ganho de altura. Os autores alertam que a parada do tratamento após a normalização da altura deve ser cuidadosamente avaliada, uma vez que a velocidade de crescimento pode diminuir de maneira significativa prejudicando o ganho final de altura.

As vantagens do tratamento contínuo ou descontínuo foram avaliadas em uma análise de quatro estudos independentes (47), dois com cada modalidade de tratamento. As crianças em tratamento contínuo receberam GH na dose de 33 ou $67 \mu \mathrm{g} / \mathrm{kg} /$ dia $(\sim 0,1$ e 0,2 $\mathrm{UI} / \mathrm{kg} /$ dia). Já as em tratamento descontínuo receberam doses mais altas de 67 a $100 \mu \mathrm{g} / \mathrm{kg} /$ dia por dois anos, ficaram, no mínimo, um ano sem tratamento e somente retomavam o tratamento se a altura voltasse a ficar abaixo de -2 DP ou se estivessem em puberdade. Foram tratadas quatro crianças a intervalos de três me- 
ses com e sem GH. Ao final de seis anos de acompanhamento, o ganho de altura foi similar entre as diferentes modalidades de tratamento. As vantagens do regime descontínuo foram o menor número de injeções e a dose menor de GH por quilo ao final dos seis anos de tratamento, uma vez que as crianças tinham peso mais baixo quando iniciaram o tratamento. Os autores alertam que as crianças nascidas PIG com deficiência de GH associada não devem ser tratadas com o regime descontínuo. Não foram discutidos os efeitos de doses altas de GH, concentrações mais elevadas de IGF-l e segurança do tratamento.

\section{Polimorfismo do receptor do GH e resposta ao tratamento}

Os modelos de previsão que utilizam variáveis clínicas, como idade, estágio puberal, altura da criança, estatura dos pais, dose e duração da terapia, não conseguem explicar satisfatoriamente a grande variabilidade na resposta ao tratamento com $\mathrm{GH}$ em crianças não deficientes de GH. Por esta razão, vários estudos têm investigado uma possível relação entre fatores genéticos e a resposta terapêutica, com ênfase em genes que participam dos mecanismos de ação do $\mathrm{GH}$. $\mathrm{O}$ gene que codifica o receptor do GH (GHR) tem sido um dos mais estudados, não só pela sua importância fisiológica, mas especialmente pelos polimorfismos estruturais observados em estudos populacionais. O principal polimorfismo do GHR consiste na deleção completa do éxon 3 (d3-GHR) que resulta da perda dos resíduos 7-28 e substituição do aminoácido A6D na porção $\mathrm{N}$-terminal do domínio extracelular do receptor (48). Em experimentos com células HEK293, Dos Santos e cols. (49) encontraram sinalização 30\% maior em células transfectadas com homodímeros e heterodímeros do d3-GHR do que nas células com o receptor completo (fl-GHR), sugerindo que a presença de d3-GHR poderia se associar com uma melhor resposta ao tratamento com GH. De fato, estes autores demonstraram que após dois anos de terapia com GH, a aceleração do crescimento foi 1,7 a 2 vezes maior nas crianças que apresentavam o polimorfismo d3-GHR. Entretanto, estudos subseqüentes em crianças com baixa estatura idiopática, nascidas PIG, deficientes de GH e em meninas com síndrome de Turner, produziram resultados conflitantes, com alguns confirmando melhor resposta na presença do d3-GHR e outros não demonstrando qualquer diferença.

A série estudada por Dos Santos e cols. era formada por um grupo heterogêneo de crianças com baixa esta- tura, incluindo somente 35\% de crianças nascidas PIG. Tauber e cols. (50), em um estudo multinacional envolvendo 240 crianças nascidas PIG, demonstraram velocidade de crescimento significativamente maior nas crianças com uma ou duas cópias do alelo d3-GHR em comparação com as portadoras do fl-GHR, tanto após o primeiro quanto após o segundo ano de tratamento. Por outro lado, não houve diferença entre os grupos em relação a mudanças na altura. Um aspecto a se destacar neste estudo foi que os autores fizeram uma análise paralela dos resultados, dividindo o grupo de acordo com a dose de GH empregada no tratamento, e demonstraram associação entre d3-GHR e resposta terapêutica somente no grupo que utilizou doses altas de GH.

Binder e cols. (51) estudaram 60 crianças nascidas PIG e não observaram ganho de velocidade significante no grupo d3-GHR após o primeiro ano de tratamento, embora este grupo tenha demonstrado crescimento mais rápido do que o previsto. Similarmente, Carrascosa e cols. (52) não conseguiram demonstrar associação entre a presença de $\mathrm{d} 3$-GHR e a resposta ao tratamento com GH em uma coorte de 86 crianças nascidas PIG que foram tratadas durante dois anos com dose de $66 \mu \mathrm{g} /$ $\mathrm{kg} /$ dia de GH. Em uma outra publicação (53), estes mesmos autores não encontraram qualquer associação entre o genótipo e o resultado terapêutico em um grupo de 60 crianças nascidas PIG que foram tratadas com doses mais baixas de $\mathrm{GH}(32,1 \mu \mathrm{g} / \mathrm{kg} /$ dia $)$, sugerindo que outros fatores, além da dose de GH usada no tratamento, levam aos resultados discrepantes entre os estudos.

Em conjunto, os dados publicados até o presente momento indicam que a influência do d3-GHR na resposta ao tratamento com GH em crianças nascidas PIG, se existente, é de pouca relevância clínica.

\section{Efeito do tratamento sobre metabolismo de lipídios e carboidratos}

$\mathrm{O}$ baixo peso ao nascimento se associa com maior risco de resistência insulínica, diabetes melito tipo 2 , hipertensão e dislipidemia na vida adulta, fatores estes diretamente vinculados ao desenvolvimento de problemas cardiovasculares (54). Pelos conhecidos efeitos do GH sobre o metabolismo de lipídeos, carboidratos e sensibilidade à insulina, há constante preocupação sobre possíveis efeitos adversos do uso prolongado de $\mathrm{GH}$ sobre estes fatores de risco, em especial no tratamento da baixa estatura de crianças nascidas PIG. No estudo de Cutfield e cols. (55), a sensibilidade à insulina (medida pelo modelo mínimo de Bergman após teste endo- 
venoso de tolerância à glicose) reduziu 44\% após 21 meses de tratamento com $\mathrm{GH}$ em um pequeno grupo de 12 crianças nascidas PIG. Uma análise individual dos pacientes deste estudo revela que em três crianças não ocorreu qualquer alteração na sensibilidade insulínica, em sete a mudança foi leve a moderada e em um caso o declínio foi pronunciado. Nos três pacientes puberais, a sensibilidade insulínica permaneceu inalterada ou levemente reduzida, ao passo que as anormalidades mais significativas ocorreram em crianças pré-puberais, sugerindo que os esteróides sexuais possa ser um fator de confusão na relação entre $\mathrm{GH}$ e resistência à insulina. Neste mesmo estudo, cinco crianças pré-púberes foram reavaliadas três meses após a suspensão do tratamento com GH e os autores não observaram recuperação da sensibilidade insulínica. Por outro lado, Sas e cols. $(56,57)$ estudaram o metabolismo de carboidratos (glicemia e insulinemia durante teste oral de tolerância à glicose e medidas de hemoglobina glicosilada), perfil lipídico, pressão arterial e composição corporal em um grupo de 79 crianças nascidas PIG (2l com DGH) antes e até seis anos após o uso de GH. Nestes estudos, o tratamento prolongado com $\mathrm{GH}$ resultou efeitos positivos sobre a composição corporal, pressão arterial e perfil lipídico, e não provocou mudanças significativas nos níveis glicêmicos e de hemoglobina glicosilada, embora tenha se associado com elevação nos níveis de insulina, tanto de jejum quanto estimulados pela glicose. Independentemente das conclusões dos estudos, todos concordam que os efeitos tardios da hiperinsulinemia provocada pelo tratamento com GH não estão totalmente estabelecidos, sendo fortemente recomendada monitoração cuidadosa durante a vida adulta de todo o paciente nascido PIG que recebeu GH para tratamento da baixa estatura $(57,58)$.

\section{CONSIDERAÇÕES FINAIS}

O tratamento com GH em crianças com baixa estatura nascidas PIG pode levar à altura adulta dentro do esperado para a população e adequada para o alvo genético. A eficácia do tratamento será maior quanto mais precoce for iniciado e quanto melhor for a altura no início da puberdade. A normalização da altura antes da puberdade é essencial. O efeito dose-dependente é evidente no início do tratamento e nas crianças pré-púberes, mas diminui com períodos mais longos de tratamento e durante a puberdade. Com base nos diferentes protocolos avaliados, uma sugestão de tratamento (59) seria uma dose de aproximadamente $33 \mu \mathrm{g} / \mathrm{kg} /$ dia $(\sim 0,1 \mathrm{UI} /$ $\mathrm{kg} /$ dia), contínuo e com correção da dose pelo ganho de peso nas crianças com altura não muito comprometida. Já nas crianças nascidas PIG com altura abaixo de -3 DP, o tratamento poderia ser iniciado com dose maior, de $50 \mu \mathrm{g} / \mathrm{kg} / \mathrm{dia}(\sim 0,15 \mathrm{UI} / \mathrm{kg} / \mathrm{dia})$, sem correção para o peso até atingir a dose de $33 \mu \mathrm{g} / \mathrm{kg} /$ dia, quando começaria a correção da dose pelo peso. Com isso, a dose mais alta no início do tratamento levaria à recuperação mais rápida no crescimento exatamente nas crianças mais comprometidas.

\section{Conflito de Interesses}

A autora MCSB é membro do Strategic Advisory Board, Pfizer International.

\section{REFERÊNCIAS}

1. Saenger P, Czernichow P, Hughes I, Reiter EO. Small for gestational age: short stature and beyond. Endocr Rev. 2007; 28(2):219-51.

2. Karlberg J, Albertsson-Wikland K. Growth in full-term smallfor-gestational-age infants: from birth to final height. Pediatr Res. 1995;38:733-9.

3. Pescador MVB, Streher AAF, Silva JMF, Valente GC, Nakagiri $\mathrm{M}$, Boguszewski MCS. Aspectos endocrinológicos das crianças e adultos nascidos pequenos para a idade gestacional. Arq Bras Encocrinol Metabol. 2001;45(1):15-23.

4. Lee PA, Chernausek SD, Hokken-Koelega AC, Czernichow P. International small for gestational age advisory board consensus development conference statement: management of short children born small for gestational age. Pediatrics. 2003;111: 1253-61.

5. Clayton PE, Cianfarani S, Czernichow P, Johannsson G, Rapaport R, Rogol A. Management of the child born small for gestational age through to adulthood: a consensus statement of the International Societies of Pediatric Endocrinology and the Growth Hormone Research Society. J Clin Endocrinol Metab. 2007;92(3):804-10.

6. Karlberg JP, Albertsson-Wikland K, Kwan EY, Lam BC, Low LC. The timing of early postnatal catch-up growth in normal, fullterm infants born short for gestational age. Horm Res. 1997;48 Suppl 1:17-24.

7. Hokken-Koelega AC, De Ridder MA, Lemmen RJ, Den Hartog H, De Muinck Keizer-Schrama SM, Drop SL. Children born small for gestational age; do they catch up? Pediatr Res. 1995;38:267-71.

8. Albertsson-Wikland K, Karlberg J. Natural growth in children born small for gestational age with and without catch-up growth. Acta Paediatr Suppl. 1994;399:64-70.

9. Chaussain JL, Colle M, Ducret JP. Adult height in children with prepubertal short stature secondary to intrauterine growth retardation. Acta Paediatr Suppl 1994;399:72-3.

10. Carrascosa A, Vicens-Calvet E, Yeste D, Espadero RM, Ulied A. Children born small for gestational age (SGA) who fail to achieve catch up growth by 2-8 years of age are short from infancy to adulthood. Data from a cross-sectional study of 486 Spanish children. Pediatr Endocrinol Rev. 2006;4(1):15-27. 
11. Preece MA. Prepubertal and pubertal endocrinology. In: Falkner $F$, Tanner JM, editors. Human growth. A comprehensive treatise. $2^{\mathrm{a}}$ ed. New York: Plenum Press; 1986. p. 211-24.

12. Carel JC, Chatelain P, Rochiccioli P, Chaussain JL. Improvement in adult height after growth hormone treatment in adolescents with short stature born small for gestational age: results of a randomized controlled study. J Clin Endocrinol Metab. 2003;88(4):1587-93.

13. Boguszewski MCS, Rosberg S, Albertsson-Wikland K. Spontaneous 24-hour growth hormone profiles in prepubertal small for gestational age children. J Clin Endocrinol Metab. 1995;80:2599.

14. Boguszewski MCS, Bjarnason R, Jansson C, Rosberg S, Albertsson-Wikland $\mathrm{K}$. Hormonal status of childern born small for gestational age. Acta Paediatr Suppl. 1997;423:189-92.

15. Ackland FM, Stanhope R, Eyre C, Hamill G, Jones J, Preece MA. Physiological growth hormone secretion in children with short stature and intra-uterine growth retardation. Horm Res. 1988; 30:241-5.

16. Albertsson-Wikland K. Growth hormone secretion and growth treatment in children with intrauterine growth retardation. Acta Paediatr Scand Suppl. 1989;349:35-41.

17. de Waal WJ, Hokken-koelega ACS, Stijnen T, de Muinck KeizerSchrama SMPF, Drop SLS. Endogenous and stimulated GH secretion, urinary GH excretion, and plasma IGF-1 and IFG-II levels in prepubertal children with short stature after intruterine growth retardation. Clin Endocrinol (Oxf). 1994;41: 621-30.

18. Boguszewski MCS, Jansson C, Rosberg S, Albertsson-Wikland $\mathrm{K}$. Changes in serum insulin-like growth factor 1 (IGF-1) and IGF-binding protein-3 levels during growth hormone treatment in prepubertal short children born small for gestational age. J Clin Endocrinol Metab. 1996;81:3902-8.

19. De Zegher F, François I, van Helvoirt M, Van den Berghe G. Small as fetus and short as child: from endogenus to exogenus growth hormone. J Clin Endocrinol Metab. 1997;82(7): 2021-6.

20. VölkI TM, Schwöbel K, Simm D, Beier C, Rohrer TR, Dörr HG. Spontaneous growth hormone secretion and IGF1:IGFBP3 molar ratios in children born small for gestational age (SGA). Growth Horm IGF Res. 2004;14(6):455-61.

21. Donaldson M. What is the role of growth-hormone therapy in short children who were small for gestational age? Lancet. 2001;358:347-8.

22. Chatelain $P$, Carrascosa $A$, Bona G, Ferrandez-Longas A, Sippell W. Growth hormone therapy for short children born small for gestational age. Horm Res. 2007;68:300-9.

23. Tanner JM, Whitehouse RH, Hughes PCR, Vince FP. Effect of human growth hormone treatment for 1 to 7 years on growth of 100 children, with growth hormone deficiency, low birthweight, inherited smallness, Turner's syndrome, and other complaints. Arch Dis Child. 1971;46:745.

24. Grunt JA, Enriquez AR, Daughaday WH. Acute and long term responses to hGH in children with idiopathic small-for-dates dwarfism. J Clin Endocrinol Metab. 1972 Jul;35(1):157-68.

25. Foley TP Jr, Thompson RG, Shaw M, Baghdassarian A, Nissley SP, Blizzard RM. Growth responses to human growth hormone in patients with intrauterine growth retardation. J Pediatr. 1974;84(5):635-41.

26. Lanes R, Plotnick LP, Lee PA. Sustained effect of human growth hormone therapy on children with intrauterine growth retardation. Pediatrics. 1979;63(5):731-5.

27. Albertsson-Wikland K. Growth hormone secretion and growth hormone treatment in children with intrauterine growth retar- dation. Swedish Paediatric Study Group for Growth Hormone Treatment. Acta Paediatr Scand Suppl. 1989;349:35-41.

28. Stanhope R, Preece MA, Hamill G. Does growth hormone treatment improve final height attainment of children with intrauterine growth retardation? Arch Dis Child. 1991;66:1180-3.

29. Chaussain JL, Colle M, Landier F. Effects of growth hormone therapy in prepubertal children with short stature secondary to intrauterine growth retardation. Acta Paediatr Suppl. 1994;399:74-5.

30. Chatelain P, Job JC, Blanchard J, Ducret JP, Oliver M, Sagnard $\mathrm{L}$, et al. Dose-dependent catch-up growth after 2 years of growth hormone treatment in intrauterine growth-retarded children. J Clin Endocrinol Metab. 1994;78:1454-60.

31. Chernausek SD, Breen TJ, Frank GR. Linear growth in response to growth hormone treatment in children with short stature associated with intrauterine growth retardation: the National Cooperative Growth Study experience. J Pediatr. 1996;128(5 Pt 2):S22-7.

32. de Zegher F, Maes M, Gargoski SE, Heinrichs C, Du Caju MV, Thiry G, et al. High-dose growth hormone treatment of short children born small for gestational age. J Clin Endocrinol Metab. 1996;81:1887-92.

33. Fjellestad-Paulsen A, Czernichow P, Brauner R, Bost M, Colle M, Lebouc JY, et al. Three-year data from a comparative study with recombinant human growth hormone in the treatment of short stature in young children with intrauterine growth retardation. Acta Paediatr. 1998;87(5):511-7.

34. Job JC, Chaussain JL, Job B, Ducret JP, Maes M, Olivier M, et al. Follow-up of three years of treatment with growth hormone and of one post-treatment year, in children with severe growth retardation of intrauterine onset. Pediatr Res. 1996; 39(2):354-9.

35. Boguszewski MCS, Albertsson-Wikland K, Aronsson S, Gustafsson J, Hagenäs L, Westgren U, et al. Growth hormone treatment of short children born small-for-gestational-age: the Nordic Multicentre Trial. Acta Paediatr. 1998;87:257-63.

36. Sas $T$, de Waal W, Mulder $P$, Houdijk $M$, Jansen $M$, Reeser $M$, et al. Growth hormone treatment in children with short stature born small for gestational age: 5-year results of a randomized, double-blind, dose-response trial. J Clin Endocrinol Metab. 1999;84(9):3064-70.

37. Argente J, Gracia R, Ibáñez L, Oliver A, Borrajo E, Vela A, et al. Improvement in growth after two years of growth hormone therapy in very young children born small for gestational age and without spontaneous catch-up growth: results of a multicenter, controlled, randomized, open clinical trial. J Clin Endocrinol Metab. 2007;92(8):3095-101.

38. Ranke MB, Lindberg A. Growth hormone treatment of short children born small for gestational age or with Silver-Russell syndrome: results from KIGS (Kabi International Growth Study), including the first report on final height. Acta Paediatr. 1996;85 Suppl 417:18-26.

39. Coutant R, Carel JC, Letrait M, Bouvattier C, Chatelain P, Coste $\mathrm{J}$, et al. Short stature associated with intrauterine growth retardation: final height of untreated and growth hormone-treated children. J Clin Endocrinol Metab. 1998;83:1070-4.

40. Zucchini S, Cacciari E, Balsamo A, Cicognani A, Tassinari D, Barbieri $E$, et al. Final height of short subjects of low birth weight with and without growth hormone treatment. Arch Dis Child. 2001;84(4):340-3.

41. Van Pareren $Y$, Mulder $P$, Houdijk $M$, Jansen $M$, Reeser $M$, Hokken-Koelega A. Adult height after long-term, continuous growth hormone $(\mathrm{GH})$ treatment in short children born small for gestational age: results of a randomized, double-blind, 
dose-response GH trial. J Clin Endocrinol Metab. 2003; 88(8):3584-90.

42. Dahlgren J, Wikland KA. Final height in short children born small for gestational age treated with growth hormone. Pediatr Res. 2005;57(2):216-22.

43. van Gool SA, Kamp GA, Visser-van Balen H, Mul D, Waelkens $\mathrm{JJ}$, Jansen $\mathrm{M}$, et al. Final height outcome after three years of growth hormone and gonadotropin-releasing hormone agonist treatment in short adolescents with relatively early puberty. J Clin Endocrinol Metab. 2007;92(4):1402-8.

44. Darendeliler F, Bundak R, Eryilmaz SK, Günöz H, Ba F, Saka N. Follow-up height after discontinuation of growth hormone treatment in children with intrauterine growth retardation. J Pediatr Endocrinol Metab. 2002;15(6):795-800

45. Fjellestad-Paulsen A, Simon D, Czernichow P. Short children born small for gestational age and treated with growth hormone for three years have an important catch-down five years after discontinuation of treatment. J Clin Endocrinol Metab. 2004;89(3):1234-9.

46. Simon D, Leger J, Fjellestad-Paulsen A, Crabbé R, Czernichow P. Intermittent recombinant growth hormone treatment in short children born small for gestational age: four-year results of a randomized trial of two different treatment regimens. Horm Res. 2006;66(3):118-23.

47. de Zegher F, Albertsson-Wikland K, Wollmann HA, Chatelain P, Chaussain JL, Löfström A, et al. Growth hormone treatment of short children born small for gestational age: growth responses with continuous and discontinuous regimens over 6 years. J Clin Endocrinol Metab. 2000;85(8):2816-21.

48. Sobrier ML, Duquesnoy P, Duriez B, Amselem S, Goossens M. Expression and binding properties of two isoforms of the human growth hormone receptor. FEBS Lett. 1993;319(1-2): 16-20.

49. Dos Santos C, Essioux L, Teinturier C, Tauber M, Goffin V, Bougnères $\mathrm{P}$. A common polymorphism of the growth hormone receptor is associated with increased responsiveness to growth hormone. Nat Genet. 2004;36(7):720-4.

50. Tauber M, Ester W, Auriol F, Molinas C, Fauvel J, Caliebe J, et al. GH responsiveness in a large multinational cohort of SGA children with short stature (NESTEGG) is related to the exon 3 GHR polymorphism. Clin Endocrinol (Oxf). 2007;67(3):457-61.

51. Binder G, Baur F, Schweizer R, Ranke MB. The d3-growth hormone $(\mathrm{GH})$ receptor polymorphism is associated with increased responsiveness to $\mathrm{GH}$ in Turner syndrome and short small-for-gestational-age children. J Clin Endocrinol Metab. 2006;91(2):659-64.

52. Carrascosa $A$, Esteban $C$, Espadero R, Fernández-Cancio $M$, Andaluz $\mathrm{P}$, Clemente $\mathrm{M}$, et al. The $\mathrm{d} 3 / \mathrm{fl}$-growth hormone (GH) receptor polymorphism does not influence the effect of $\mathrm{GH}$ treatment $(66 \mathrm{microg} / \mathrm{kg}$ per day) or the spontaneous growth in short non-GH-deficient small-for-gestational-age children: results from a two-year controlled prospective study in 170 Spanish patients. J Clin Endocrinol Metab. 2006;91(9):3281-6.

53. Carrascosa A, Audí L, Esteban $C$, Fernández-Cancio $M$, Andaluz $\mathrm{P}$, Gussinyé $\mathrm{M}$, et al. Growth hormone (GH) dose, but not exon 3-deleted/full-length $\mathrm{GH}$ receptor polymorphism genotypes, influences growth response to two-year GH Therapy in Short Small-for-Gestational-Age Children. J Clin Endocrinol Metab. 2008;93(1):147-53.

54. Barker DJ, Hales CN, Fall CH, Osmond C, Phipps K, Clark PM. Type 2 (non-insulin-dependent) diabetes mellitus, hypertension and hyperlipidaemia (syndrome $\mathrm{X}$ ): relation to reduced fetal growth. Diabetologia. 1993;36(1):62-7.

55. Cutfield WS, Jackson WE, Jefferies C, Robinson EM, Breier $\mathrm{BH}$, Richards GE, et al. Reduced insulin sensitivity during growth hormone therapy for short children born small for gestational age. J Pediatr. 2003;142(2):113-6.

56. Sas TC, de Muinck Keizer-Schrama SM, Stijnen T, Aanstoot HJ, Drop SL. Carbohydrate metabolism during long-term growth hormone $(\mathrm{GH})$ treatment and after discontinuation of $\mathrm{GH}$ treatment in girls with Turner syndrome participating in a randomized dose-response study. Dutch Advisory Group on Growth Hormone. J Clin Endocrinol Metab. 2000;85(2):769-75.

57. Sas T, Mulder P, Aanstoot HJ, Houdijk M, Jansen M, Reeser M, et al. Carbohydrate metabolism during long-term growth hormone treatment in children with short stature born small for gestational age. Clin Endocrinol (Oxf). 2001;54(2):243-51.

58. Silverstein JH, Shulman D. Growth hormone for small-forgestational-age children: short and sweet? J Pediatr. 2003 142(2):91-2.

59. de Zegher F, Hokken-Koelega A. Growth hormone therapy for children born small for gestational age: height gain is less dose dependent over the long term than over the short term. Pediatrics. 2005;115(4):e458-62.

Endereço para correspondência:

Margaret C. S. Boguszewski

Unidade de Endocrinologia Pediátrica

Rua Padre Camargo, 250

80060-240 Curitiba, PR

E-mail: margabogus@uol.com.br 